\title{
Positive Homogeneity of the Principle of Equivalent Utility
}

\author{
J. Chudziak, M. Chudziak, B. Sobek
}

Faculty of Mathematics and Natural Sciences, University of Rzeszów, Prof. S. Pigonia 1, 35-310 Rzeszów, Poland

\begin{abstract}
We prove that if the principle of equivalent utility under the cumulative prospect theory is positively homogeneous on a relatively small family of risks for every non-negative initial wealth level, then a value function is linear for gains and losses, but, in general, it needs not be linear.
\end{abstract}

DOI: 10.12693/APhysPolA.129.941

PACS/topics: 89.65.Gh

\section{Introduction}

Assume that $(\Omega, \Sigma, P)$ is a probability space and $L^{\infty}(\Omega, \Sigma, P)$ is a linear space of all bounded random variables on $(\Omega, \Sigma, P)$. Let $\Delta \subset L^{\infty}(\Omega, \Sigma, P)$ be a family of non-negative random variables, describing the risks which are covered by an insurance company. Assume that the company has a continuous and strictly increasing utility function $u: \mathbb{R} \rightarrow \mathbb{R}$ and the initial wealth $w$. Using the properties of $u$, it is not difficult to show that, for every $X \in \Delta$, there exists a unique real number $H_{w}(X)$ such that

$$
u(w)=E u\left(w+H_{w}(X)-X\right) .
$$

In this way, Eq. (1) defines a functional $H_{w}: \Delta \rightarrow \mathbb{R}$, called the principle of equivalent utility (under the expected utility theory). It is important for the theoretical as well as for the practical reasons and, in fact, it is one of the most frequently studied premium principles. In the case where $w=0$, the principle of equivalent utility is called the zero utility principle.

In a paper by Kałuszka and Krzeszowiec [1], the principle of equivalent utility under the cumulative prospect theory has been investigated. This approach leads to the equation

$$
u(w)=E_{g h}\left(u\left(w+H_{w}(X)-X\right)\right),
$$

where $E_{g h}$ is a generalized Choquet integral with respect to the probability distortion functions $g$ and $h$ for gains and losses, respectively and $u: \mathbb{R} \rightarrow \mathbb{R}$ is a value function. Let us recall that for a given distortion function $g:[0,1] \rightarrow[0,1]$, that is a non-decreasing function with $g(0)=0$ and $g(1)=1$, and a random variable $X \in L^{\infty}(\Omega, \Sigma, P)$, the Choquet integral related to $g$ is defined in the following way:

$$
\begin{aligned}
& E_{g}(X)=\int_{-\infty}^{0}(g(P(X>t))-1) \mathrm{d} t \\
& \quad+\int_{0}^{\infty} g(P(X>t)) \mathrm{d} t .
\end{aligned}
$$

Several details concerning the Choquet integral and its properties can be found in [2]. The generalized Choquet integral, related to the distortion functions $g$ (for gains) and $h$ (for losses), is defined as follows:

$$
E_{g h}(X)=E_{g}(\max \{X, 0\})-E_{h}(\max \{-X, 0\})
$$

$$
\text { for } X \in L^{\infty}(\Omega, \Sigma, P) \text {. }
$$

In Ref. [1], among others, the positive homogeneity of the principle of equivalent utility has been considered. Assuming that $a X \in \Delta$ for $X \in \Delta, a>0$, a premium principle $H$ is said to be positively homogeneous (or scale invariant) on the family $\Delta$, provided $H(a X)=a H(X)$ for $X \in \Delta$ and $a>0$. Let us introduce the following notation. For every $x>0$ and $p \in[0,1]$, by $(x, p)$ we denote the random variable $X$ such that $P(X=0)=1-p$ and $P(X=x)=p$. Furthermore, we set

$$
\mathcal{X}_{2}:=\{(x, p) \mid x>0, p \in[0,1]\} .
$$

In Ref. [1] (cf. Theorem 5) it has been proved that if $u: \mathbb{R} \rightarrow \mathbb{R}$ is a continuous and strictly increasing value function with $u(0)=0, g$ and $h$ are continuous probability distortion functions for gains and losses, respectively such that, for every $p \in\left(p_{0}, 1\right)$, with some fixed $p_{0} \in(0,1)$, it holds

$$
g(1-p) h(p)>0
$$

and the premium $H_{0}$ is positively homogeneous on $\mathcal{X}_{2}$, then

$$
u(x)= \begin{cases}-b(-x)^{r} & \text { for } \quad x<0, \\ a x^{r} & \text { for } \quad x \geq 0\end{cases}
$$

with some $a, b, r>0$. Conversely, if for some fixed $p_{0} \in(0,1)$ and for every $p \in\left(p_{0}, 1\right)$, (5) holds and $u$ is of the form (6) with some $a, b, r>0$, then the premium $H_{0}$ is positively homogeneous on $L^{\infty}(\Omega, \Sigma, P)$. Furthermore, if the premiums $H_{0}$ and $H_{w}$ for some $w>0$, are positively homogeneous on $\mathcal{X}_{2}$, then

$$
h(p)=1-g(1-p) \quad \text { for } \quad p \in(0,1)
$$

and

$$
u(x)=\alpha x \quad \text { for } \quad x \in \mathbb{R}
$$

with some $\alpha>0$. Conversely, if (7) holds and $u$ is of the form (8) with some $\alpha>0$, then, for every $w \geq 0$, the premium $H_{w}$ is positively homogeneous on $L^{\infty}(\Omega, \Sigma, P)$. These results have been generalized by Chudziak et al. [3], who proved that if

$$
H_{0}\left(a_{i} X\right)=a_{i} H_{0}(X) \quad \text { for } \quad X \in \mathcal{X}_{2}, i \in\{1,2\}
$$

where $a_{1}, a_{2} \in(0, \infty) \backslash\{1\}$ are such that $\frac{\ln a_{1}}{\ln a_{2}}$ is irrational, then $u$ is of the form (6) with some $a, b, r>0$. If, additionally,

$$
H_{w}\left(a_{i} X\right)=a_{i} H_{w}(X) \text { for } \quad X \in \mathcal{X}_{2}, i \in\{1,2\},
$$


where $w>0$ is fixed, then (7) and (8) hold. According to the methods applied in the proofs of the above results, a positive homogeneity has to be satisfied on $\mathcal{X}_{2}$. From a formal point of view, this assumption requires the property $\{P(A): A \in \Sigma\}=[0,1]$ which, in fact, is not explicitly expressed in the papers (it is known that every non-atomic probability measure has such a property). On the other hand, as $\mathcal{X}_{2}$ consists of the risk $(x, p)$, where $p \in[0,1]$ and $x>0$ are arbitrary, from a practical point of view, it could be difficult to check, if a positive homogeneity holds on the whole $\mathcal{X}_{2}$. It seems to be reasonable to assume that a positive homogeneity holds for as small family of risk as possible and to derive from such an assumption the forms of the premium and the value function. Following this idea, in our paper we deal with the positive homogeneity of the premium of equivalent utility on the family of risks

$$
\mathcal{X}_{2, p}:=\{(x, p) \mid x>0\},
$$

where $p \in(0,1)$ is fixed. Therefore, formally we need only to assume that the probability measure $P$ is nontrivial, that is it takes at least one value from the interval $(0,1)$. Practically, the family $\mathcal{X}_{2, p}$ can describe, for example, the risk of a car accident, where $p$ is a known probability of an accident and $x$ is a value of loss.

It is remarkable that the positively homogeneous premiums of equivalent utility under the expected utility and under the rank-dependent utility have been studied by Reich [4] and Heilpern [5], respectively.

\section{Results}

Note that, if $X$ is a random variable, taking the values $x$ and $y$ with probabilities $P(X=x)=q$ and $P(X=$ $y)=1-q$, where $x, y \in \mathbb{R}, q \in[0,1]$, then, applying (3) and (4), after a standard computation, we get

$$
\begin{gathered}
E_{g h}(X)=(1-g(1-q)) x+g(1-q) y \\
\text { whenever } \quad 0 \leq x<y, \\
E_{g h}(X)=(1-h(q)) y+h(q) x \\
\text { whenever } \quad x<y \leq 0
\end{gathered}
$$

and

$$
\begin{gathered}
E_{g h}(X)=g(1-q) y+h(q) x \\
\text { whenever } \quad x<0<y .
\end{gathered}
$$

The following theorem is a main result of the paper.

Theorem 2.1 Assume that $p \in(0,1)$ is fixed, $\mathcal{X}_{2, p}$ is given by (9), $g$ and $h$ are probability distortion functions for gains and losses, respectively such that (5) holds and $u: \mathbb{R} \rightarrow \mathbb{R}$ is a continuous and strictly increasing value function with $u(0)=0$. Then the following two statements are equivalent:

(i) for every $w \geq 0, H_{w}$ is a positively homogeneous premium of equivalent utility on the family $\mathcal{X}_{2, p}$;

(ii) there exists an $\alpha>0$ such that

$$
u(x)= \begin{cases}\frac{1-g(1-p)}{h(p)} \alpha x & \text { for } \quad x<0 \\ \alpha x & \text { for } \quad x \geq 0\end{cases}
$$

and

$$
\begin{aligned}
& H_{w}((x, p))=(1-g(1-p)) x \\
& \quad \text { for } \quad w \geq 0, x>0 .
\end{aligned}
$$

Proof. Assume that $(i)$ holds. Then

$$
H_{w}((a x, p))=a H_{w}((x, p)) \quad \text { for } \quad w \geq 0, a, x>0 .
$$

Setting in this equality $x=1$, we get

$$
H_{w}((a, p))=c(w) a \quad \text { for } \quad w \geq 0, a>0,
$$

where a function $c:[0, \infty) \rightarrow \mathbb{R}$ is given by $c(w)=$ $H_{w}((1, p))$ for $w \geq 0$. Since a generalized Choquet integral is monotone and $E_{g h}(c)=c$ for $c \in \mathbb{R}$ (see Lemma 1 in [1]), taking into account the fact that $u$ is strictly increasing, in view of (15), we obtain

$$
\begin{aligned}
& u(w+c(w) x-x)=E_{g h} u\left(w+H_{w}((x, p))-x\right) \leq \\
& E_{g h} u\left(w+H_{w}((x, p))-(x, p)\right) \leq \\
& E_{g h} u\left(w+H_{w}((x, p))\right)=E_{g h} u(w+c(w) x)= \\
& u(w+c(w) x) \text { for } \quad w \geq 0, x>0 .
\end{aligned}
$$

Furthermore, from (2) it follows that:

$$
\begin{aligned}
& E_{g h} u\left(w+H_{w}((x, p))-(x, p)\right)=u(w) \\
& \quad \text { for } \quad w \geq 0, x>0 .
\end{aligned}
$$

Hence

$$
\begin{aligned}
& u(w+c(w) x-x) \leq u(w) \leq u(w+c(w) x) \\
& \quad \text { for } \quad w \geq 0, x>0
\end{aligned}
$$

and so, as $u$ is strictly increasing, we conclude that

$$
w+c(w) x-x \leq w \leq w+c(w) x \quad \text { for } \quad w \geq 0, x>0 .
$$

This means that

$$
c(w) \in[0,1] \text { for } w \geq 0 .
$$

Suppose that $c(w)=1$ for some $w \geq 0$. Then, in view of (15), we have $H_{w}((x, p))=x$ for $x>0$. Therefore, for every $x>0$, a random variable $u\left(w+H_{w}((x, p))-(x, p)\right)$ takes two non-negative values $u(w), u(w+x)$ with probabilities $p$ and $1-p$, respectively. Therefore, as $u(w)<$ $u(w+x)$ for every $x>0$, applying (10), from (16) we derive

$$
\begin{aligned}
& (1-g(1-p)) u(w)+g(1-p) u(w+x)=u(w) \\
& \quad \text { for } \quad x>0 .
\end{aligned}
$$

Hence

$$
g(1-p)(u(w+x)-u(w))=0 \quad \text { for } \quad x>0
$$

which, in view of $(5)$, gives a contradiction. In this way we have proved that $c(w) \neq 1$ for $w \geq 0$. Therefore, taking into account (17), we conclude that

$$
c(w) \in[0,1) \text { for } w \geq 0 .
$$

We show that 
$c(0) \neq 0$.

For the proof by contradiction, suppose that $c(0)=0$. Then, in virtue of (15), we get $H_{0}((x, p))=0$ for $x>0$. Hence, for every $x>0$, a random variable $u\left(H_{0}((x, p))-(x, p)\right)$ takes two non-positive values $u(-x)$ and $u(0)=0$ with probabilities $p$ and $1-p$, respectively. Since $u(-x)<0$ for $x>0$, making use of (11), from (16) we derive that $h(p) u(-x)=0$ for $x>0$. As $u$ is strictly increasing, $u(0)=0$ and (5) holds, this yields a contradiction. Therefore, (19) is proved.

According to (15) and (18), for every $w \geq 0$ and $x>$ $\frac{w}{1-c(w)}$, a random variable $u\left(w+H_{w}((x, p))-(x, p)\right)$ takes a negative value $u(w+(c(w)-1) x)$ with a probability $p$ and a positive value $u(w+c(w) x)$ with a probability $1-p$. Hence, making use of (2) and (12), we get

$$
\begin{aligned}
& g(1-p) u(w+c(w) x)+h(p) u(w+(c(w)-1) x)=u(w) \\
& \quad \text { for } \quad w \geq 0, x>\frac{w}{1-c(w)} .
\end{aligned}
$$

Setting in (20) $w=0$, we obtain

$$
\begin{aligned}
& g(1-p) u(c(0) x)+h(p) u((c(0)-1) x)=0 \\
& \quad \text { for } \quad x>0 .
\end{aligned}
$$

Since, in view of (18), $\frac{x}{c(0)-1}>0$ for $x<0$, replacing in this equality $x$ by $\frac{x}{c(0)-1}$ and making use of (5), we get

$$
u(x)=-\frac{g(1-p)}{h(p)} u\left(\frac{c(0)}{c(0)-1} x\right) \quad \text { for } \quad x<0 .
$$

Furthermore, as $w+(c(w)-1) x<0$ for $w \geq 0$ and $x>\frac{w}{1-c(w)}$, taking into account (20) and (22), for every $w \geq 0$ and $x>\frac{w}{1-c(w)}$, we obtain

$$
\begin{aligned}
& g(1-p) u(w+c(w) x)-g(1-p) \\
& \quad \times u\left(\frac{c(0)}{c(0)-1}(w+(c(w)-1) x)\right)=u(w) .
\end{aligned}
$$

Since $\frac{w-\frac{c(0)-1}{c(0)} z}{1-c(w)}>\frac{w}{1-c(w)}$ for $w \geq 0, z>0$, inserting into the last equality $x=\frac{w-\frac{c(0)-1}{c(0)} z}{1-c(w)}$, we obtain

$$
\begin{aligned}
& u\left(\frac{1-c(0)}{c(0)} \frac{c(w)}{1-c(w)} z+\frac{w}{1-c(w)}\right)=u(z)+\frac{u(w)}{g(1-p)} \\
& \quad \text { for } \quad w \geq 0, z>0 .
\end{aligned}
$$

As $u$ is continuous, passing in (24) to the limit with $z \rightarrow$ $0^{+}$, we get

$$
\frac{u(w)}{g(1-p)}=u\left(\frac{w}{1-c(w)}\right) \quad \text { for } \quad w \geq 0 .
$$

Therefore, (24) becomes

$$
\begin{aligned}
& u\left(\frac{1-c(0)}{c(0)} \frac{c(w)}{1-c(w)} z+\frac{w}{1-c(w)}\right)=u(z) \\
& \quad+u\left(\frac{w}{1-c(w)}\right) \text { for } w, z \geq 0 .
\end{aligned}
$$

Now, we are going to prove that $c$ is continuous on $[0, \infty)$. Suppose that this is not true, that is $c$ is discontinuous at some $w_{0} \geq 0$. Since, in view of (18), $c$ is a bounded function, this means that there exists a sequence $\left(w_{n}\right)$ of positive real numbers such that $\lim _{n \rightarrow \infty} w_{n}=w_{0}$ and a sequence $\left(c\left(w_{n}\right)\right)$ is convergent to a limit different from $c\left(w_{0}\right)$. Put $d:=\lim _{n \rightarrow \infty} c\left(w_{n}\right)$. Then $d \neq c\left(w_{0}\right)$ and, by (18), $d \in[0,1]$. Moreover, according to (25), for every $n \in \mathbb{N}$, we have

$$
\frac{u\left(w_{n}\right)}{g(1-p)}=u\left(\frac{w_{n}}{1-c\left(w_{n}\right)}\right) .
$$

Letting in this equality $n \rightarrow \infty$, using the continuity of $u$ and applying (25) again, we obtain

$$
u\left(\frac{w_{0}}{1-c\left(w_{0}\right)}\right)=\lim _{n \rightarrow \infty} u\left(\frac{w_{n}}{1-c\left(w_{n}\right)}\right) .
$$

If $d \neq 1$ then using the continuity of $u$, in view of (27), we get

$$
u\left(\frac{w_{0}}{1-c\left(w_{0}\right)}\right)=u\left(\frac{w_{0}}{1-d}\right) .
$$

Since $u$ is strictly increasing, this implies that $d=c\left(w_{0}\right)$, which yields a contradiction. If $d=1$ and $w_{0}>0$, then $\lim _{n \rightarrow \infty} \frac{w_{n}}{1-c\left(w_{n}\right)}=\infty$, which contradicts $(27)$, as $u$ is strictly increasing. Finally, if $d=1$ and $w_{0}=0$ then from (27) we derive that $\lim _{n \rightarrow \infty} \frac{w_{n}}{1-c\left(w_{n}\right)}=0$. Therefore, as $u$ is continuous, setting in (26) $w=w_{n}$ for $n \in \mathbb{N}$ and letting $n \rightarrow \infty$, we obtain

$$
\begin{aligned}
& \lim _{n \rightarrow \infty} u\left(\frac{1-c(0)}{c(0)} \frac{c\left(w_{n}\right)}{1-c\left(w_{n}\right)} z+\frac{w_{n}}{1-c\left(w_{n}\right)}\right)=u(z) \\
& \quad \text { for } \quad z \geq 0 .
\end{aligned}
$$

On the other hand, we have

$$
\lim _{n \rightarrow \infty}\left(\frac{1-c(0)}{c(0)} \frac{c\left(w_{n}\right)}{1-c\left(w_{n}\right)} z+\frac{w_{n}}{1-c\left(w_{n}\right)}\right)=\infty
$$$$
\text { for } z \geq 0 \text {, }
$$

which again yields a contradiction. In this way we have proved that $c$ is continuous.

Define the functions $a, b:[0, \infty) \rightarrow \mathbb{R}$ in the following way:

$$
\begin{aligned}
& a(w)=\frac{1-c(0)}{c(0)} \frac{c(w)}{1-c(w)} \text { for } w \in[0, \infty), \\
& b(w)=\frac{w}{1-c(w)} \text { for } w \in[0, \infty) .
\end{aligned}
$$

Obviously, as $c$ is continuous, so are $a$ and $b$. Furthermore, according to (25) and (29), we have

$$
b(w)=u^{-1}\left(\frac{u(w)}{g(1-p)}\right) \quad \text { for } \quad w \geq 0 .
$$

This means that $b$ is injective. Moreover, $b(0)=0$ and, making use of (18), we get $\lim _{w \rightarrow \infty} b(w)=\infty$. Therefore, $b$ is a bijection on $[0, \infty)$. Note also that from $(26)-(29)$ it follows that:

$$
u(a(w) z+b(w))=u(z)+u(b(w)) \text { for } \quad w, z \geq 0 .
$$

Hence

$$
f(F(w) z+w)=f(z) f(w) \text { for } \quad w, z \geq 0,
$$

where a function $f: \mathbb{R} \rightarrow \mathbb{R}$ is given by

$$
f(x)=e^{u(x)} \quad \text { for } \quad x \in \mathbb{R}
$$

and $F: \mathbb{R} \rightarrow \mathbb{R}$ is an arbitrary continuous extension of 
$\left(a \circ b^{-1}\right)$ from $[0, \infty)$ onto $\mathbb{R}$. In particular, we have

$$
F(x)=\left(a \circ b^{-1}\right)(x) \text { for } x \geq 0 .
$$

Equation (30) is the Goląb-Schinzel type functional equation on $[0, \infty)$. Since $f$ and $F$ are continuous, $f$ is strictly increasing and $f(x)>0$ for $x \in \mathbb{R}$, applying Theorem 3.4 in Ref. [6], we conclude that one of the following two possibilities holds:

1. there exist $\alpha, r>0$ such that $f(x)=(1+\alpha x)^{r}$ for $x \geq 0$ and

$$
F(w)=1+\alpha w \quad \text { for } \quad w \geq 0 ;
$$

2. $F=1$ and there exists an $\alpha>0$ such that

$$
f(x)=e^{\alpha x} \quad \text { for } \quad x \geq 0 .
$$

In the first case, making use of (28), (29), (32), and (33), we get

$$
\begin{aligned}
& \frac{1-c(0)}{c(0)} \frac{c(w)}{1-c(w)}=a(w)=F(b(w))=1+\frac{\alpha w}{1-c(w)} \\
& \quad \text { for } w \geq 0 .
\end{aligned}
$$

Hence

$$
c(w)=c(0)(1+\alpha w) \text { for } \quad w \geq 0
$$

and so, in view of (19), we obtain $\lim _{w \rightarrow \infty} c(w)=\infty$, which contradicts (18).

If the second possibility holds then, by (32), we have $a(w)=1$ for $w \geq 0$ which, together with (28), gives

$$
c(w)=c(0) \quad \text { for } \quad w \geq 0 .
$$

Furthermore, (31) and (34) imply that $u(x)=\alpha x$ for $x \geq 0$. Thus, taking into account (22), we get

$$
u(x)=\frac{c(0) g(1-p)}{(1-c(0)) h(p)} \alpha x \quad \text { for } \quad x<0 .
$$

Consequently, putting $\beta:=\frac{c(0) g(1-p)}{(1-c(0)) h(p)} \alpha$, we conclude that $\beta>0$ and

$$
u(x)=\left\{\begin{array}{l}
\beta x \text { for } \quad x<0, \\
\alpha x \text { for } \quad x \geq 0 .
\end{array}\right.
$$

Inserting into (20) $u$ of the form (36) and making use of (35), for every $w \geq 0$ and $x>\frac{w}{1-c(0)}$, we obtain

$$
\begin{aligned}
& (g(1-p) \alpha+h(p) \beta-\alpha) w+(g(1-p) \alpha c(0) \\
& \quad+h(p) \beta(c(0)-1)) x=0 .
\end{aligned}
$$

Hence

$$
g(1-p) \alpha c(0)+h(p) \beta(c(0)-1)=0
$$

and

$$
g(1-p) \alpha+h(p) \beta-\alpha=0 .
$$

Multiplying both sides of (38) by $c(0)$ and subtracting obtained in this way equality from (37), we get

$$
h(p)=c(0) \frac{\alpha}{\beta} .
$$

From (37) and (39) we derive that

$$
g(1-p)=1-c(0) \text {. }
$$

Therefore, making use of (15) and (35), we obtain (14). Furthermore, (39) and (40) imply that $\beta=\frac{1-g(1-p)}{h(p)} \alpha$ which, together with (36), gives (13). Consequently, (ii) is valid.

A straightforward calculation shows that if $u$ is of the form (13) with some $\alpha>0$ and (14) holds then, for every $w \geq 0, H_{w}$ is a positively homogeneous premium of equivalent utility on the family $\mathcal{X}_{2, p}$. Thus, $(i i)$ implies $(i)$, which completes the proof.

Corollary 2.2 Assume that $L^{\infty}(\Omega, \Sigma, P)$ is a probability space such that $\{P(A): A \in \Sigma\}=[0,1], g$ is a continuous at 0 probability distortion functions for gains, $h$ is a continuous at 1 probability distortion functions for losses and there exists a sequence $\left(p_{n}\right)$ of elements of the interval $(0,1)$ such that $\lim _{n \rightarrow \infty} p_{n}=1$ and $g\left(1-p_{n}\right) h\left(p_{n}\right)>0$ for $n \in \mathbb{N}$. If, for every $w \geq 0$, the premium $H_{w}$ is positively homogeneous on the family $\bigcup_{n \in \mathbb{N}} \mathcal{X}_{2, p_{n}}$, then

$$
h\left(p_{n}\right)=1-g\left(1-p_{n}\right) \quad \text { for } \quad n \in \mathbb{N}
$$

and $u$ is of the form (8) with some $\alpha>0$.

Proof. Assume that, for every $w \geq 0$, the premium $H_{w}$ is positively homogeneous on the family $\bigcup_{n \in \mathbb{N}} \mathcal{X}_{2, p_{n}}$. Applying Theorem 2.1, we conclude that there is an $\alpha>0$ such that, for every $n \in \mathbb{N}$, it holds

$$
u(x)=\left\{\begin{array}{lll}
\frac{1-g\left(1-p_{n}\right)}{h\left(p_{n}\right)} \alpha x & \text { for } & x<0, \\
\alpha x & \text { for } & x \geq 0 .
\end{array}\right.
$$

Since $g$ is continuous at $0, h$ is continuous at $1, g(0)=0$ and $h(1)=1$, letting in (42) $n \rightarrow \infty$, we get (8). Furthermore, (8) and (42) imply (41).

\section{Conclusion}

Inspired by the results in [1], we have considered the positively homogeneous premium of equivalent utility under the cumulative prospect theory. We have proved that if the probability distortion functions for gains and losses satisfy some natural assumptions and for every non-negative wealth level, the premium is positively homogeneous on a relatively small family of risks, then the value function is linear for gains and losses, but, in general, it is not linear.

\section{References}

[1] M. Kałuszka, M. Krzeszowiec, Insur. Math. Econ. 50, 159 (2012).

[2] D. Denneberg, Lectures on Non-Additive Measure and Integral, Kluwer, Boston 1994.

[3] J. Chudziak, M. Halicki, S. Wójcik, Acta Phys. Pol. A 127, A-29 (2015).

[4] A. Reich, ASTIN Bull. 14, 123 (1984).

[5] S. Heilpern, Insur. Math. Econ. 33, 67 (2003).

[6] J. Chudziak, Z. Kočan, Results Math. 66, 199 (2014). 